Pacific Journal of Mathematics

ON SUPPORTS OF REGULAR BORER MEESSLRES 


\title{
ON SUPPORTS OF REGULAR BOREL MEASURES
}

\author{
D. J. Hebert and H. Elton Lacey
}

The existence of a regular Borel measure whose support is a given compact Hausdorff space $X$ imposes definite structures on $X, C(X)$, and $C(X)^{*}$. In this paper a necessary and sufficient condition is given to insure that $X$ is the support of a regular Borel measure. This involves the intersection number of a collection of open sets in $X$. Measures which vanish on a sigma ideal of a sigma field of subsets of $X$ which contains a basis for the topology of $X$ are also considered. In particular, for a certain class of compact Hausdorff spacs $X$, necessary and sufficient conditions are given to insure the existence of a nonatomic regular Borel measure whose support is $X$. The final section of the paper is devoted to a study of normal measures; i.e., measures which vanish on meager Borel sets. Normal measures on $X$ are shown to be related to normal measures on the projective resolution of $X$.

Notation AND Terminology. Set theoretical and topological terminology is that of [12], the terminology of linear topological spaces is that of [14], and measure theory terminology follows [11]. All spaces considered are taken to be nonempty and all measures considered are finite. If $X$ is a compact Hausdorff space, $C(X)$ denotes the space of continuous real-valued functions on $X$ in the supremum norm, $C(X)^{*}$ denotes the space of all continuous linear functionals on $C(X)$, or, equivalently, the space of all signed regular Borel measures, and $B(X)$ denotes the space of all bounded real-valued functions on $X$ in the supremum norm.

1. Intersection numbers. The following definitions are motivated by the concept of an intersection number as given in [13]. Let $X$ be a compact Hausdorff space and $B$ be a Boolean algebra.

1.1. If $S=\left(f_{1}, \cdots, f_{n}\right)$ is a finite sequence in $B(X), i(S)=$ $(1 / n)\left\|\sum_{\imath=1}^{n} f_{i}\right\|$. If $A \subseteq C(X)$, then $I(A)=\inf \{i(S): S$ is a finite sequence in $A\}$.

1.2. If $S=\left(A_{1}, \cdots, A_{n}\right)$ is a finite sequence of subsets of $X$, $i(S)=\max \left\{(k / n)\right.$ : there is a subsequence $\left(A_{i_{1}}, \cdots, A_{i_{k}}\right)$ of $S$ such that $\left.\bigcap_{j=1}^{k} A_{i}, \neq \varnothing\right\}$. If $H$ is a collection of subsets of $X$, then $I(H)=$ $\inf \{i(S): S$ is a finite sequence in $H\}$.

1.3. If $S=\left(E_{1}, \cdots, E_{n}\right)$ is a finite sequence in $B$, then $i(S)=$ 
$\max \left\{(k / n)\right.$ : there is a subsequence $\left(E_{i_{1}}, \cdots, E_{i_{n}}\right)$ of $S$ such that $\left.\bigwedge_{j=1}^{k} E_{i}, \neq 0\right\}$ and $I(H)=\inf \{i(S): S$ is a finite sequence in $H\}$.

The relationship between the above concepts is the following: if $Y$ is the Stone space of $B$ (see [10] or [18]), then for $H \subseteq B, I(H)=$ $I\{h(E): E \in H\}=I\left\{C_{h(E)}: E \in H\right\}$, where $h$ is the isomorphism of $B$ onto the clopen (i.e., closed and open) sets of $Y$ and $C_{h(E)}$ is the characteristic function of $h(E)$ (this notation for the characteristic function is used throughout).

The numbers $I(A)$ and $I(H)$ above are called the intersection numbers of the collections $A$ and $H$ respectively.

Lemma 1.4. Let $X$ be a compact Hausdorff space and let $F$ and $G$ be nonempty subsets of the positive cone of $C(X)$. If $a>0$ is such that, for each $f \in F$, there is a $g \in G$ with $a g \leqq f$, then $a$ $I(G) \leqq I(F)$.

Proof. Let $S=\left(f_{1}, \cdots, f_{n}\right)$ be a finite sequence in $F$. For each $i, 1 \leqq i \leqq n$, there is a $g_{i}$ in $G$ such that $a g_{i} \leqq f_{i}$. Thus

$$
0 \leqq \sum_{i=1}^{n} a g_{i} \leqq \sum_{i=1}^{n} f_{i} \text { and }\left\|\sum_{i=1}^{n} a g_{i}\right\| \leqq\left\|\sum_{i=1}^{n} f_{i}\right\| \text {. }
$$

If $T=\left(g_{1}, \cdots, g_{n}\right)$, then

$$
a I(G) \leqq a i(T)=a\left(\frac{1}{n}\right)\left\|\sum_{i=1}^{n} g_{i}\right\|=\left(\frac{1}{n}\right)\left\|\sum_{i=1}^{n} a g_{i}\right\| \leqq\left(\frac{1}{n}\right)\left\|\sum_{i=1}^{n} f_{i}\right\|=i(S) .
$$

Hence $a I(G) \leqq I(F)$.

Definition 1.5. Let $B$ be a Boolean algebra and let $H$ be a nonempty subset of $B$. Then $H$ is said to be positive if and only if $I(H)>0$. Similarly, if $H$ is a nonempty collection of nonempty subsets of a given set, then $H$ is said to be a positive collection whenever $I(H)>0$.

THEOREm 1.6. If $X$ is a compact Hausdorff space with topology $G$, then there is a regular Borel measure whose support is $X$ if and only if $G \backslash\{\phi\}$ is the union of a countable family of positive collections. (See [13], Th. 4)

Proof. Suppose $G \backslash\{\phi\}$ is the union of a countable family $\left\{G_{n}\right\}$ of positive collections. For each $f$ in $C(X)$ and each $u$, let

$$
A_{f}=\left\{x \in X: f(x)>\frac{1}{2}\right\}
$$


and $F_{n}=\left\{f \in C(X): f \geqq 0,\|f\|=1\right.$, and $A_{f}$ is in $\left.G_{n}\right\}$, and denote the convex hull of $F_{n}$ by $Q_{n}$. It is a matter of computation to show that $\|f\| \geqq I\left(F_{n}\right)$ for each $f \in Q_{n}$ and that for each $f \in F_{n},(1 / 2) C_{A_{f}} \leqq f$. By Lemma $1.4,0<(1 / 2) I\left(G_{n}\right) \leqq I\left(F_{n}\right)$, and by the above, $\|f\| \geqq I\left(F_{n}\right)>0$ for all $f \in Q_{n}$. There is a positive linear functional $\dot{\phi}_{n}$ on $C(X)$ such that $\phi_{n}(f) \geqq I\left(F_{n}\right)$ for all $f \in Q_{n}$. For, if $U=\left\{g \in C(X):\|g\|<I\left(F_{n}\right)\right\}$, then $Q_{n}+U$ is open and convex and $\left(Q_{n}+U\right) \cap-P=\varnothing$, where $P=f \in C(X) \mid f \geqq 0\}$. By [14], p. 118, there is a continuous linear functional $\dot{\phi}_{n}$ on $C(X)$ such that $\dot{\phi}_{n}(-f) \leqq \phi_{n}(g+h)$ for each $f \in P$, $g \in Q_{n}$, and $h \in U$. Since 0 is in $-P, 0 \leqq \phi_{n}(g+h)$ for all $g \in Q_{n}$, and $h \in U$. Thus $\phi_{n}$ is a positive linear functional and, without loss of generality, it is assumed that $\left\|\phi_{n}\right\|=1$. Suppose $g \in Q_{n}, I\left(F_{n}\right)>\varepsilon>0$, and let 1 denote the constant function 1 on $X$. Then $\left(\varepsilon-I\left(F_{n}\right)\right) 1$ is in $U$, and $0 \leqq \phi_{n}\left[g+\left(\varepsilon-I\left(F_{n}\right)\right) 1\right]=\dot{\phi}_{n}(g)-I\left(F_{n}\right) \dot{\phi}_{n}(1)+\varepsilon \dot{\phi}_{n}(1)=$ $\dot{\rho}_{n}(g)-I\left(F_{n}\right)+\varepsilon$ and hence, $\dot{\phi}_{n}(g) \geqq I\left(F_{n}\right)-\varepsilon$. Let $\phi=\sum_{n=1}^{\infty}\left(1 / 2_{n}\right) \dot{\phi}_{n}$. Then $\phi$ is a positive linear functional on $C(X)$ and $\|\phi\|=1$. Now, suppose $F \in P$ and $f \neq 0$. Then $g=f /\|f\|$ has norm 1 and thus, $g \in F_{n}$ for some $n$. Hence, $\dot{\phi}(g) \geqq \dot{\phi}_{n}(g) \geqq I\left(F_{n}\right)>0$. It follows immediately that the regular Borel measure that corresponds to $\dot{\phi}$ has support all of $X$.

Conversely, suppose $X$ has a regular Borel measure whose support is $X$. Then there is a positive normalized linear functional $\phi$ on $X$ such that $\phi(f)>0$ if $f \geqq 0, f \neq 0$. Let $F=\{f \in C(X) \mid f \geqq 0,\|f\|=1\}$ and $F_{n}=\{f \in F \mid \dot{\phi}(f)>(1 / n)\}$. By computation if follows that

$$
\frac{1}{n} \leqq \inf \left\{\dot{\phi}(f): f \in F_{n}\right\} \leqq I\left(F_{n}\right) .
$$

For each $f \in F$ and each $n$, let $B_{f}=\{x \in X: f(x)>0\}$ and $H_{n}=$ $\left\{B_{f}: f \in F_{n}\right\}$. From Urysohn's lemma it follows that for each open subset $U$ of $X$ there is an $f \in F$ such that $f(x)=0$ for $x \notin U$ and $f(x)=1$ for some $x \in U$ and thus, $f \leqq C_{B_{f}} \leqq C_{U}$. If $G_{n}=\{T \in G: U$ contains a member of $\left.H_{n}\right\}$, then for each $U$ in $G_{n}$ there is an $f \in F_{n}$ such that $f \leqq C_{B_{f}} \leqq C_{U}$. By Lemma 1.4, $0<I\left(F_{n}\right) \leqq I\left(H_{n}\right) \leqq I\left(G_{n}\right)$. Clearly $G \backslash\{\phi\}=\bigcup_{n=1}^{\infty} G_{n}$.

Corollary 1.7. If $X$ is a compact Hausdorff space and $B$ is a basis for the topology $G$ of $X$, then there is a regular Borel measure whose support is $X$ if and only if $B \backslash\{\dot{\phi}\}$ is the union of A countable family of positive collections.

2. Nonatomic measures. In this section, certain conditions are shown to be sufficient for the existence of a nonatomic regular Borel measure whose support is a given compact Hausdorff space. The 
study of supports of nonatomic regular Borel measures is shown to be related to the study of perfect separable compact Hausdorff spaces.

If $m$ is a regular Borel measure on a compact Hausdorff space $X$, then $m$ is called nonatomic if for each $x$ in $X, m(\{x\})=0$.

Proposition 2.1. [17] If $X$ is a compact Hausdorff space, then there is a nonzero, nonatomic regular Borel measure on $X$ if and only if $X$ has a nonempty perfect subset.

A well-known topological lemma is also needed.

Lemma 2.2. If $C$ is a closed subset of a topological space $X$, then $\operatorname{int} C=\operatorname{int} \operatorname{cl} \operatorname{int} C$.

THEOREM 2.3. If $X$ is a perfect compact Hausdorff space such that there exists a regular Borel measure $m$ on $X$ whose support is $X$, then there are perfect subsets $X_{1}$ and $X_{2}$ of $X$ such that

(i) either $X_{1}$ is empty or there is a nonatomic regular Borel measure whose support is $X_{1}$,

(ii) either $X_{2}$ is empty or $X_{2}$ is separable,

(iii) $X=X_{1} \cup X_{2}$,

(iv) int $X_{1}$ contains $X \backslash X_{2}$ and int $X_{2}$ contains $X \backslash X_{1}$.

Proof. Suppose $C=\left\{x_{1}, x_{2}, \cdots\right\}=\{x$ in $X: m(\{x\})>0\}$ and $m=$ $m_{1}+m_{2}$ where $m_{1}$ is nonatomic and $m_{2}=\sum_{n=1}^{\infty} m\left(\left\{x_{n}\right\}\right) e_{x_{n}}$. Let $X_{1}=$ $\operatorname{Supp}\left(m_{1}\right)$ and let $X_{2}=\mathrm{cl}$ int $\operatorname{Supp}\left(m_{2}\right)$. Since $m_{1}\left(X \backslash \operatorname{Supp}\left(m_{1}\right)\right)=0$ and $m_{2}\left\lfloor X \backslash \operatorname{Supp}\left(m_{2}\right)\right]=0$, if $A=X \backslash \operatorname{Supp}\left(m_{1}\right) \cap X \backslash \operatorname{Supp}\left(m_{2}\right)$, then

$$
0=m_{1}(A)=m_{2}(A)=m(A) .
$$

Since the complement of the support of a regular Borel measure is open and since the $m$-measure of a nonempty open set is positive, $A$ is open; therefore empty. It follows that $\operatorname{Supp}\left(m_{2}\right)$ contains the open set $X \backslash \operatorname{Supp}\left(m_{1}\right)$ : hence, int Supp $\left(m_{2}\right)$ contains $X \backslash X_{1}$, and $X_{2}$ contains $X \backslash X_{1}$. Therefore, $X_{1} \cup X_{2}=X$. Also, cl $X_{2}$ contains cl $X \backslash X_{1}$, and hence int $X_{1}=X \backslash \mathrm{cl} X \backslash X_{1}$ contains $X \backslash \mathrm{cl} X_{2}=X \backslash X_{2}$. Since int $X_{2}$ is int cl int Supp $\left(m_{2}\right)$, by 2.2 int $X_{2}=\operatorname{int} \operatorname{Supp}\left(m_{2}\right)$. Hence, int $X_{2}$ contains $X \backslash X_{1}$. Suppose that $x$ is in $X_{2}$ and $U$ is an open set subset of $X$ containing $x$. Since $X_{2}=\mathrm{cl}$ int $\operatorname{Supp}\left(m_{2}\right), U \cap \operatorname{int} \operatorname{Supp}\left(m_{2}\right)$ is nonempty. It is easy to see that $\operatorname{Supp}\left(m_{2}\right)=\mathrm{cl} C$; hence, there is a member $y$ of $C$ such that $y$ is also an element of the open set $U \cap \operatorname{int} \operatorname{Supp}\left(m_{2}\right)$. This implies that $X_{2}$ is separable, $\left(C \cap X_{2}\right.$ is a countable dense subset). If $x$ is not in int $\operatorname{Supp}\left(m_{2}\right)$, then $y \neq x$. If $x$ is in int Supp $\left(m_{2}\right)$, then since $X$ is perfect, there is a member of 
$U \cap \operatorname{int} \operatorname{Supp}\left(m_{2}\right)$ which is distinct from $x$. Hence, $X_{2}$ is perfect.

CoROLlaRY 2.4. If $X$ is a compact Hausdorff space on which there is a regular Borel measure whose support is $X$, and such that each countable subset of $X$ is nowhere dense, then there is a nonatomic regular Borel measure on $X$ whose support is $X$.

Proof. First note that since each singleton is nowhere dense, $X$ is perfect. Continuing the notation of the preceding theorem, since $\operatorname{Supp}\left(m_{2}\right)=\mathrm{cl} C$, and $C$ is nowhere dense, int $\operatorname{Supp}\left(m_{2}\right)$ is empty, and hence $X_{2}$ is empty. This implies that $X=X_{1}$, and the proof is finished.

According to Theorem 2.3, the problem of the existence of a nonatomic regular Borel measure whose support is a given compact Hausdorff space $X$, which has a measure whose support is $X$, may be reduced to the same problem for a separable perfect compact Hausdorff space. The theorem also makes possible the development of other sufficient conditions on such spaces via the following corollary.

CoROllaRY 2.5. If $X$ is a perfect compact Hausdorff space with a regular Borel measure whose support is $X$, and such that each point of $X$ is contained in the support of a nonatomic regular Borel measure, then there is a nonatomic regular Borel measure whose support is $X$.

Proof. Suppose that $X_{1}, X_{2}$, and $m_{1}$ are chosen as in the theorem. If $X_{2}$ is nonempty, let $\left\{x_{1}, x_{2}, \cdots\right\}$ be a countable dense subset of $X_{2}$. For each $k$, let $\nu_{k}$ be a nonatomic regular Borel measure whose support contains $x_{k}$. Then $\nu=\sum_{n=1}^{\infty}\left(1 / 2^{k}\right) \nu_{k}$ is a nonatomic regular Borel measure whose support is $X_{2}$, and $m_{1}+\nu$ is a nonatomic regular regular Borel measure whose support is $X$.

Lemma 2.6. If $X$ is a perfect compact Hausdorff space, and $x$ is an element of $X$ such that there is a countable base of open neighborhoods of $x$, then there is a nonatomic regular Borel measure whose support contains $x$.

Proof. Let $\left\{U_{1}, U_{2}, \cdots\right\}$ be a countable base of open neighborhoods of $x$. For each $k, U_{k}$ contains a perfect subset $P_{k}$, which by (2.1) contains the support of a nonatomic regular Borel measure $\nu_{k}$. Let $\nu=\sum_{n=1}^{\infty}\left(1 / 2^{k}\right) \nu_{k}$. If $U$ is an open set containing $x$, then $U$ contains $U_{k}$ for some $k$; hence, $U$ contains $P_{k}$, and $\nu_{k}(U)>0$. 
THEOREM 2.7. If $X$ is a perfect compact Hausdorff space which satisfies the first axiom of countability, and there is a regular Borel measure whose support is $X$, then there is a nonatomic regular Borel measure whose support is $X$.

Proof. It follows immediately from the preceding results.

COROLLARY 2.8. If $X$ is a perfect compact metrizable Hausdorff space, then there is a nonatomic regular Borel measure whose support is $X$.

3. J-null measures. Let $B$ be a Boolean sigma-algebra, $J$ be a sigma-ideal in $B$, and $G$ be a subset of $B$. Using some of the methods in [13], conditions may be obtained for the existence of a measure on $B$ which vanishes on $J$ and which is bounded away from zero on $G$. A set $G$ which satisfies such conditions is called $J$-sigmapositive. Let $B$ be a sigma-field of subsets of a topological space, and let $J$ be a sigma-ideal in $B$ containing no open sets. If $B$ contains a basis $G$ of open sets, then there is a measure on $B$, which vanishes on $J$ and is positive on nonempty open sets if and only if $G \backslash\{\phi\}$ is contained in a countable union of $J$-sigma-positive collections. This result is used along with results of $\S 2$ to give necessary and sufficient conditions for the existence of a nonatomic regular Borel measure on a class of compact Hausdorff spaces $X$ whose support is all of $X$.

Let $B$ be a Boolean algebra and let $h$ be the natural isomorphism of $B$ onto the Boolean algebra of clopen subsets of the Stone space $Y$ of $B$. The following lemma are basic results in [13].

Lemma 3.1. If $G$ is a positive subset of $B$, then there is a finitely additive measure $m$ on $B$ such that $m(E) \geqq I(G)$ for each $E$ in $G$.

Lemma 3.2. If $m$ is a finitely additive measure on $B$ and $G$ is $a$ subset of $B$, then $\inf \{m(E): E$ is in $G\} \leqq I(G)$.

Definition 3.3. If $B$ is a Boolean sigma-algebra and $C$ is a subset of $B$, then $C$ is said to be a monotone class whenever for each monotone sequence $E_{1} \leqq E_{2} \leqq \cdots$ of elements of $C ; \mathrm{V}_{n=1}^{\infty} E_{n}$ is an element of $C$.

Definition 3.4. Let $B$ be a Boolean sigma-algebra, $G$ be a subset of $B$, and $\hat{G}=\{E$ in $B: D \leqq E$ for some $D$ in $G\}$. The collection $G$ is said to be sigma-positive if and only if $G$ is positive and $B \backslash \widehat{G}$ is a monotone class. 
The following construction of a measure on a sigma-algebra is based on an idea of Ryll-Nardzewski (see [13]).

Lemma 3.5. If $B$ is a sigma-algebra and $G$ is a sigma-positive subset of $B$, then there is a measure $m$ on $B$ such that $m(E) \geqq I(G)$ for each $E$ in $G$.

Proof. By 3.1, there is a finitely additive measure $m^{\prime}$ on $B$ such that $m^{\prime}(E) \geqq I(G)$ for each $E$ in $G$. For each $E$ in $B$, let $m(E)=$ $\inf \left\{\sum_{n=1}^{\infty} m^{\prime}\left(A_{n}\right): A_{1}, A_{2}, \cdots\right.$ is a pairwise disjoint sequence such that $\left.\bigvee_{n=1}^{\infty} A_{n}=E\right\}$. Clearly, $0 \leqq m(E) \leqq m^{\prime}(E)$ for each $E$ in $B$. Let $A_{1}, A_{2}, \cdots$ be a pairwise disjoint sequence in $B$. If $\varepsilon$ is a positive number, then for each $n$, there is a pairwise disjoint sequence $C_{1}^{n}, C_{2}^{n}, \cdots$ such that $A_{n}=\mathbf{V}_{k=1}^{\infty} C_{k}^{n}$ and

$$
m\left(A_{n}\right) \leqq \sum_{k=1}^{\infty} m^{\prime}\left(C_{k}^{n}\right)<m\left(A_{n}\right)+\left(\varepsilon / 2^{n}\right) .
$$

Hence,

$$
\sum_{n=1}^{\infty} m\left(A_{n}\right) \leqq \sum_{n=1}^{\infty} \sum_{k=1}^{\infty} m^{\prime}\left(C_{k}^{n}\right)<\sum_{n=1}^{\infty} m\left(A_{n}\right)+\varepsilon .
$$

Since $\mathbf{V}_{n=1}^{\infty} A_{n}=\mathbf{V}_{n=1}^{\infty} \bigvee_{k=1}^{\infty} C_{k}^{n}$, by the definition of $m, m\left(\mathbf{V}_{n=1}^{\infty} A_{n}\right) \leqq$ $\sum_{n=1}^{\infty} m\left(A_{n}\right)$. If $m\left(\mathbf{V}_{n=1}^{\infty} A_{n}\right)<\sum_{n=1}^{\infty} m\left(A_{n}\right)$, then there is a pairwise disjoint sequence $E_{1}, E_{2}, \cdots$ of elements of $B$ such that $\bigvee_{n=1}^{\infty} A_{n}=$ $\bigvee_{k=1}^{\infty} E_{k}$ and $m\left(\bigvee_{n=1}^{\infty} A_{n}\right) \leqq \sum_{k=1}^{\infty} m^{\prime}\left(E_{k}\right)<\sum_{n=1}^{\infty} m\left(A_{n}\right)$. Since for each

$$
n, \bigvee_{k=1}^{\infty} E_{k} \wedge A_{n}=A_{n} \wedge \bigvee_{k=1}^{\infty} E_{k}=A_{n}
$$

(see [10], p. 28), $m\left(A_{n}\right) \leqq \sum_{k=1}^{\infty} m^{\prime}\left(E_{k} \wedge A_{n}\right)$. Hence,

$$
\sum_{n=1}^{\infty} m\left(A_{n}\right) \leqq \sum_{n=1}^{\infty} \sum_{k=1}^{\infty} m^{\prime}\left(E_{k} \wedge A_{n}\right) .
$$

However, since for each $i$ and $k, \bigvee_{n=1}^{i} E_{k} \wedge A_{n} \leqq E_{k}$, it follows that

$$
\sum_{n=1}^{i} m^{\prime}\left(E_{k} \wedge A_{n}\right)=m^{\prime}\left(\sum_{n=1}^{i}\left[E_{k} \wedge A_{n}\right]\right) \leqq m^{\prime}\left(E_{k}\right) \text {. }
$$

Thus,

$$
\sum_{n=1}^{\infty} m^{\prime}\left(E_{k} \wedge A_{n}\right) \leqq m^{\prime}\left(E_{k}\right)
$$

and

$$
\sum_{n=1}^{\infty} \sum_{k=1}^{\infty} m^{\prime}\left(E_{k} \wedge A_{n}\right) \leqq \sum_{k=1}^{\infty} m^{\prime}\left(E_{k}\right)<\sum_{n=1}^{\infty} m\left(A_{n}\right) \leqq \sum_{n=1}^{\infty} \sum_{k=1}^{\infty} m^{\prime}\left(E_{k} \wedge A_{n}\right)
$$


which is a contradiction. It has been shown then that $m$ is countably additive. Suppose now that $m(E)<I(G)$ for some $E$ in $G$. There is a monotone sequence $E_{1} \leqq E_{2} \leqq \cdots$ such that $\bigvee_{n=1}^{\infty} E_{n}=E$, and $\left\{\sup \left\{m^{\prime}\left(E_{n}\right): n \geqq 0\right\}<I(G)\right.$, (this is a trivial alteration of the definition of $m$ ). Thus, for each $n, m^{\prime}\left(E_{n}\right)<I(G)$. This implies that for each $n, E_{n}$ is not in the set $\hat{G}=\{E$ in $B: D \leqq E$ for some $D$ in $G\}$. Since $B \backslash \hat{G}$ is a monotone class, $E$ is $B \backslash \hat{G}$, which is a contradiction.

DEFINITION 3.6. Let $B$ be a Boolean sigma-algebra, $J$ be a sigmaideal in $B$, and $G$ be a subset of $B$. For each $E$ in $B$, let $\bar{E}$ denote the equivalence class modulo $J$ to which $E$ belongs. The collection $G$ is said to be $J$-sigma-positive whenever $\{\bar{E}: E$ is in $G\}$ is a sigmapositive subset of $B / J$.

Proposition 3.7. Let $B$ be a sigma-algebra and let $J$ be a sigmaideal in $B$. Let $\bar{E}$ denote the equivalence class modulo $J$ of $E$ for each $E$ in $B$. If $m$ is a measure on $B$ such that $m(E)=0$ for each $E$ in $J$, and $\bar{m}(\bar{E})=m(E)$ for each $E$ in $B$, then $\bar{m}$ is a measure on $B / J$. Conversely, if $\bar{m}$ is a measure on $B / J$, and $m(E)=\bar{m}(\bar{E})$ for each $E$ in $B$, then $m$ is a measure on $B$ such that $m(E)=0$ for each $E$ in $J$.

The above proposition is well known. The proof is a trivial alteration of a proof in [10], p. 65 .

Definition 3.8. If $B$ is a Boolean sigma-algebra, $J$ is a sigmaideal in $B$ and $m$ is a measure on $B$, then $m$ is called $J$-null whenever $m(E)=0$ for each $E$ in $J$.

Corollary 3-9. If $B$ is a sigma-algebra, $J$ is a sigma-ideal in $B$ and $G$ is a J-sigma-positive subset of $B$, then there is a J-null measure on $B$ and a positive real number a such that $m(A) \geqq a$ for each $A$ in $G$.

Proof. Let $\bar{G}=\{\bar{E}: E$ is in $G\}$. Then $\bar{G}$ is a sigma-positive subset of $B / J$. By 3.5 , there is a measure $\bar{m}$ on $B / J$ such that $\bar{m}(\bar{E}) \geqq I(\bar{G})$ for each $E$ in $G$. Let $A=I(\bar{G})$ and let $m(E)=\bar{m}(\bar{E})$ for each $E$ in $B$. By 3.6, $\bar{m}$ is a measure on $B$ which vanishes on $J$. If $E$ is in $G$, then $\bar{E}$ is in $\bar{G}$ and $m(E)=\bar{m}(\bar{E}) \geqq a$.

TheOREM 3.10. Let $X$ be a topological space, $B$ be a sigma-field of subsets of $X$ which contains a basis $G$ of the topology of $X$, and $J$ be a sigma-ideal in $B$ which contains no open sets. Then there is a J-null measure $m$ on $B$ such that $m(U)>0$ for each nonempty 
open subsets $U$ of $X$ if and only if $G \backslash\{\dot{\phi}\}$ is contained in the union of a countable family of J-sigma-positive sub-collections of $B$.

Proof. Suppose that $m$ is a $J$-null measure on $B$ which is positive on $G \backslash\{\dot{\phi}\}$. For each $n$, let $B_{n}=\{E$ in $B: m(E)>(1 / n)\}$. For each $E$ in $B$, let $\bar{E}$ denote the equivalence class modulo $J$ of $E$. Let $\bar{B}_{n}=$ $\left\{\bar{E}: E\right.$ is in $\left.B_{n}\right\}$. By 3.7, if $\bar{m}(\bar{E})=m(E)$ for each $E$ in $B$, then $\bar{m}$ is a measure on $B / I$. By $3.2,(1 / n) \leqq \inf \bar{m}(\bar{E}): \bar{E}$ is in $\bar{B}_{n} \leqq I\left(\bar{B}_{n}\right)$. Thus, $\bar{B}_{n}$ is positive. Let $\bar{E}_{1} \leqq \bar{E}_{2} \leqq \cdots$ be a monotone sequence of elements of $B / I$ such that for each $k$, and for each $\bar{E}$ in $\bar{B}_{n}$, it is not true that $\bar{E} \leqq \bar{E}_{k}$. Then for each $k, \bar{m}\left(\bar{E}_{k}\right) \leqq(1 / n)$, which implies

$$
\bar{m}\left(\sum_{k=1}^{\infty} \bar{E}_{k}\right) \leqq(1 / n),
$$

and hence it is not true that $\bar{E} \leqq \bigcup_{k=1}^{\infty} \bar{E}_{k}$ for some $\bar{E}$ in $\bar{B}_{n}$. Therefore, $\bar{B}_{n}$ is sigma-positive, and $B_{n}$ is $J$-sigma-positive.

Conversely, if $\bigcup_{n=1}^{\infty} B_{n}$ contains $G \backslash\{\dot{\phi}\}$ where for each $n, B_{n}$ is $J$ sigma-positive, then for each $n$, there is a measure $\bar{m}_{n}$ on $B / J$ such that $\bar{m}_{n}(\bar{E}) \geqq I\left(\bar{B}_{n}\right)>0$ for each $\bar{E}$ in $\bar{B}_{n}$. Let $\bar{m}=\sum_{n=1}^{\infty}\left(1 / 2^{n}\right) \bar{m}_{n}$. Then for each $\bar{U}$ in $\bar{G} \backslash\{0\}, \bar{m}(\bar{U})>0$. If $m(E)=\bar{m}(\bar{E})$ for each $E$ in $B$, then $m$ vanishes on $J$ and $m$ is a positive on $G \backslash\{\dot{\phi}\}$.

Corollary 3.11. Let $X$ be a compact Hausdorff space, $B$ be the sigma-field of Baire subsets of $X$, and $J$ be a sigma-ideal in $B$ which contains no open sets. There is a regular Borel measure with support $X$ such that $m(E)=0$ for each $E$ in $J$ if and only if the collection $G$ of nonempty open Baire subsets of $X$ is contained in the union of a countable family of J-sigma-positive collections of Baire sets.

Proof. According to $3.10, G$ is contained in the union of a countable family of $J$-sigma-positive subcollection if and only if there is a Baire measure $m$ on $X$ such that $m(U)>0$ for each open Baire set $U$ and $m(E)=0$ for each $E$ in $J$. The regular Borel extension of $m$ is the regular Borel measure required.

The final theorem of this section combines the preceding results with those of $\S 2$.

THEOREM 3.13. Let $X$ be a perfect compact Hausdorff space with topology $G$ and with the property that each point contained in an open separable subset of $X$ is a $G_{i}$-point. Let $J$ be the sigma-ideal of Borel sets which contains no nonempty perfect subsets. There is a nonatomic regular Borel measure whose support is $X$ if and only if $G$ is contained in a countable union of J-sigma-positive collections 
of Borel sets.

Proof. Suppose that $G$ is contained in a countable union of $J$ sigma-positive collections. By 3.10, there is a $J$-null Borel measure $m^{\prime}$ on $X$ such that $m^{\prime}(U)>0$ for each nonempty open subset $U$ of $X$. Let $m^{\prime \prime}$ be the restriction of $m^{\prime}$ to the Baire sets, and let $m$ be the regular Borel extension of $m^{\prime \prime}$. Then $m$ is a regular Borel measure which vanishes on Baire sets which contain no nonempty perfect subsets. Let $X_{1}, X_{2}, m_{1}, m_{2}$ be as in 2.3. Suppose that $X_{2}$ is nonempty. Then $X_{2}$ is separable and since $X_{2}=\operatorname{cl}$ int Supp $\left(m_{2}\right)$, int $X_{2}$ is nonempty. It is clear then that int $X_{2}$ is separable and locally compact in the relative topology. Thus, $\{x\}$ is a $G_{j}$ for each $x$ in int $X_{2}$, and hence $\{x\}$ is a Baire set for each $x$ in int $X_{2}$. Therefore, $m(\{x\})=0$ for each $x$ in int $X_{2}$. If $U$ is an open subset of $X \backslash X_{1}$, then since int $X_{2}$ contains $X \backslash X_{1}$, int $X_{2}$ contains $U$. Since $m(\{x\})=0$ for each $x$ in $U, m_{2}(U)=0$. Since $U \cap \operatorname{Supp} m_{2}$ is empty, $m_{1}(U)=0$. Thus, $m(U)=0$, which contradicts the fact that $m$ is positive on nonempty open Baire sets. Therefore, $X_{2}$ is empty, and $X_{1}=\operatorname{Supp}\left(m_{1}\right)=X$.

Conversely, if $m$ is a nonatomic regular Borel measure whose support is $X$, then $m$ is a $J$-null Borel measure which is positive on nonempty open Borel sets. By 3.10, $G \backslash\{\phi\}$ is contained in the union of a countable family of $J$-sigma-positive subcollections.

4. Normal measures and the projective resolution. This section develops relationships between the regular Borel measures on a compact Hausdorff space $X$ and the regular Borel measures on the projective resolution of $X$.

In [8], Gleason introduces the concept of the projective resolution of a compact Hausdorff space. If $P$ is a compact Hausdorff space, then $P$ is extremally disconnected if and only if the closure of each open subset of $P$ is open. If $X$ and $Y$ are compact Hausdorff spaces, then a continuous irreducible map of $X$ onto $Y$ is a continuous function $f$ from $X$ onto $Y$ such that $Y$ is not the image under $f$ of a proper closed subset of $X$. Gleason's result states: If $X$ is a compact Hausdorff space, then there is an extremally disconnected compact Hausdorff space $P$ (called the projective resolution of $X$ ) and a continuous irreducible map $g$ (called the Gleason map) of $P$ onto $X$, and if $P^{\prime}$ is an extremally disconnected compact Hausdorff space such that $X$ is the image of $P^{\prime}$ under a continuous irreducible map $g^{\prime}$, then there is a homeomorphism $h$ of $P$ onto $P^{\prime}$ such that $g^{\prime} h=g$.

If $X$ is a topological space and $U$ is an open subset of $X$, then $U$ is called a regular open subset of $X$ if and only if $U=\operatorname{int} \operatorname{cl} U$.

Notation. In this section, if $Y$ is a compact Hausdorff space, 
then $R(Y)$ denotes the collection of regular open subsets of $Y, B_{0}(Y)$ denotes the sigma-algebra of Borel subsets of $Y$, and $I(Y)$ denote the sigma-ideal of meager (i.e., contained in a countable union of nowhere dense sets) Borel subsets of $Y$.

If $Y$ is a compact Hausdorff space, then the collection $R(Y)$ is a Boolean algebra with the following operations: If $U$ and $V$ are in $R(X)$, then $U \vee V=\operatorname{int} \operatorname{cl}(U \cup V), U \wedge V=U \cap V$, and the complement of $U$ is $Y \backslash \operatorname{cl} U ; 0=\phi$ and $1=X$ (for a proof, see [10]). It is useful to note that each Borel subset of $Y$ is equivalent modulo $I(Y)$ to a unique regular open set, and indeed $R(Y)$ is isomorphic to $B_{0}(Y) / I(Y)$, (see $[10]$, p. 58).

In Gleason's construction, the projective resolution $P$ of a compact Hausdorff space $X$ turns out to be the Stone space of $R(X)$. The following sequence of lemmas throws some light upon the relationships involved.

Lemma 4.1. If $P$ and $X$ are compact Hausdorff spaces and $g$ is a continuous irreducible map of $P$ onto $X$, then for each nonempty open subset $U$ of $P, g(U)$ has nonempty interior.

Proof. Since $U$ is nonempty, $P \backslash U$ is a proper closed subset of $P$ and by the irreducibility of $g, g(P \backslash U)$ is a proper closed subset of $X$. If $x$ is in $X \backslash g(P \backslash U), p$ is in $P$, and $g(p)=x$, then $p$ is not in $P \backslash U$; hence $p$ is in $U$ and $x$ is in $g(U)$. Therefore, $g(U)$ contains the nonempty open set $X \backslash g(P \backslash U)$.

In the remainder of this section, $X$ is a compact Hausdorff space, $P$ is the projective resolution of $X$, and $g$ is the Gleason map of $P$ onto $X$.

Lemma 4.2. If $C$ is a nonempty clopen subset of $P$, then int $g^{-1}[g(C)]=C \quad$ and $\quad \operatorname{cl} g^{-1}[\operatorname{int} g(C)]=C$.

Proof. Since $P$ is extremally disconnected and $g(C)$ is closed, int $g^{-1}[g(C)]$ is clopen. If $D=\operatorname{int} g^{-1}[(C)] \backslash C$ is nonempty, then $P \backslash D$ is a proper clopen subset of $P$ containing $C$, and $g(P \backslash D)=X$, contradicting the irreducibility of $g$. Further, $g^{-1}[g(C)]$ contains the open set $g^{-1}[\operatorname{int} g(C)]$, and hence, $C=\operatorname{int} g^{-1}[(C)]$ contains $g^{-1}[\operatorname{int} g(C)]$.

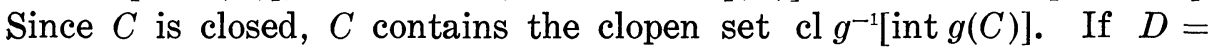

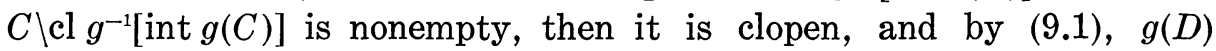
nonempty interior. But since $D$ is disjoint from $g^{-1}[\operatorname{int} g(C)], g(D)$ is contained in $g(C) \backslash$ int $g(C)$. 


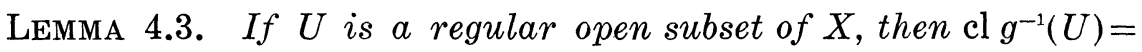

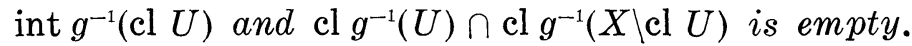

Proof. Since $g^{-1}(U)$ is a subset of $g^{-1}(\mathrm{cl} U)$, the clopen set $\operatorname{cl} g^{-1}(U)$ is a subset of the closed set $g^{-1}(\mathrm{cl} U)$, hence, $\operatorname{cl} g^{-1}(U)$ is a subset of int $g^{-1}(\mathrm{cl} U)$. Let

$$
D=\operatorname{int} g^{-1}(\mathrm{cl} U) \backslash \operatorname{cl} g^{-1}(U) .
$$

If $D$ is nonempty, then $g(D)$ is contained in $\operatorname{cl}(U) \backslash U$ which is a nowhere dense set, that $g(D)$ has nonempty interior by 4.1. Suppose

$$
\operatorname{cl} g^{-1}(U) \cap \operatorname{cl} g^{-1}(X \backslash \operatorname{cl} U)=\operatorname{int} g^{-1}[\operatorname{cl}(U)] \cap \operatorname{int} g^{-1}[\operatorname{cl}(X \backslash \operatorname{cl} U)]=A
$$

is nonempty. Since $A$ is clopen, $g(A)$ has nonempty interior, and since $g\left[\right.$ int $\left.g^{-1}(\operatorname{cl} U)\right]$ is a subset of $g\left[g^{-1}(\operatorname{cl} U)\right]=\operatorname{cl} U$ and

$$
g\left[\operatorname{int} g^{-1}(\operatorname{cl}(X \backslash \operatorname{cl} U))\right]
$$

is a subset of $\operatorname{cl}(X \backslash \mathrm{cl} U), g(A)$ is a subset of $\operatorname{cl}(U) \cap \operatorname{cl}(X \backslash \operatorname{cl} U)$, a nowhere dense set.

Lemma 4.4. If $A$ is a subset of $X$, then $A$ is nowhere dense if and only if $g^{-1}(A)$ is nowhere dense.

Proof. If $A$ is nowhere dense, then so is $\mathrm{cl} A$. If $g^{-1}(\mathrm{cl} A)$ has nonempty interior, then by (4.1), $g\left[g^{-1}(\mathrm{cl} A)\right]=\operatorname{cl} A$ has nonempty interior. Hence, $g^{-1}(\mathrm{cl} A)$ is nowhere dense and contains $g^{-1}(A)$. Conversely, if $D$ is a nowhere dense subset of $P$, then so is $\operatorname{cl} D$. Suppose that cl $g(D)$ contains a nonempty open set $V$. Then $g^{-1}(V)$ is a nonempty open subset of $g^{-1}[\mathrm{cl} g(D)]$ which is contained in $g^{-1}[g(\operatorname{cl} D)]$. Since cl $D$ contains no nonempty open set, $g^{-1}(V) \backslash$ cl $D$ contains a nonempty clopen set $C$. Since $g(\mathrm{cl} D)$ contains $V$ which contains $g(C), P \backslash g(C)$ maps onto $X$, which is a contradiction. Hence, cl $g(D)$ is nowhere dense, and $g(D)$ is nowhere dense.

LeMma 4.5. If for each regular open subset $U$ of $X, h(U)=$ cl $g^{-1}(U)$, then $h$ is an isomorphism of the Boolean algebra $R(X)$ onto the Boolean algebra of clopen subsets of $P$.

Proof. Since $P$ is extremally disconnected and $g^{-1}(U)$ is open, cl $g^{-1}(U)$ is clopen. If $C$ is a nonempty clopen subset of $P$, then $g(C)$ is closed, and by 2.2 , int $g(C)$ is a regular open set. By 4.2,

$$
h[\operatorname{int} g(C)]=\operatorname{cl~} g^{-1}[\operatorname{int} g(C)]=C .
$$

Thus, $h$ is an onto map. If $U$ and $V$ are nonempty regular open subsets of $X$ such that $h(U)=h(V)$, then suppose that $U \neq V$. Then 
either $U \cap(X \backslash \mathrm{cl} V)$ or $V \cap(X \backslash \mathrm{cl} U)$ is nonempty (otherwise, cl $U$ contains $V$, cl $U$ contains cl $V, U=$ int cl $U$ contains int cl $V=V$, and likewise $V$ contains $U)$. Suppose $W=U \cap(X \backslash \mathrm{cl} V)$ is nonempty. Then $W$ is a regular open set, and $h(W)$ is a nonempty subset of both $h(U)$ and $h(X \backslash \mathrm{cl} V)$. But $h(U)=h(V)$, and by 4.3,

$$
h(V) \cap h(X \backslash \mathrm{cl} V)
$$

is empty, which is contradictory. Therefore, $h(U)=h(V)$ and $h$ is one-to-one. To show that $h$ preserves complementation, it is necessary only to show that for each regular open set $V, h(V) \cup h(X \backslash \mathrm{cl} V)=P$. If $V$ is a nonempty regular open proper subset of $X$, then

$$
\begin{aligned}
\operatorname{cl} g^{-1}(V) \cup \operatorname{cl} g^{-1}(X \backslash \operatorname{cl} V) & =\operatorname{cl}\left[g^{-1}(V) \cup g^{-1}(X \backslash \operatorname{cl} V)\right] \\
& =\operatorname{cl}\left[g^{-1}(V \cup X \backslash \operatorname{cl} V)\right] .
\end{aligned}
$$

Since $X \backslash(V \cup X \backslash \mathrm{cl} V)$ is nowhere dense,

$$
g^{-1}(X \backslash[V \cup(X \backslash \mathrm{cl} V)])=P \backslash g^{-1}[V \cup(X \backslash \mathrm{cl} V)]
$$

is nowhere dense, and the desired result follows. Suppose that $U$ and $V$ are nonempty regular open subsets of $X$. If

$$
A=h(U \cap V)=\operatorname{cl} g^{-1}(U \cap V)=\operatorname{cl}\left[g^{-1}(U) \cap g^{-1}(V)\right],
$$

then clearly both $h(U)$ and $h(V)$ contain $A$. Let $D$ be a clopen subset of both $h(U)$ and $h(V)$. Then $h^{-1}(D)$ is contained in both $h^{-1}[h(U)]$ and $h^{-1}[h(V)]$. Thus $U \cap V$ contains $h^{-1}(D)$ and $A=h(U \cap V)$ contains $D$. Therefore, $A=h(U) \cap h(V)$. Therefore, $h$ is one-to-one, onto, and $h$ perserves complementation and intersection; thus, $h$ is an isomorphism.

THEOREM 4.6. There is a regular Borel measure whose support is $X$ if and only if there is a regular Borel measure whose support is $P$.

Proof. If $\mu$ is a regular Borel measure whose support is $P$, then $\nu(A)=\mu\left(g^{-1}(A)\right)$ defines a regular Borel measure whose support is $X$, where $g$ is the continuous irreducible map of $P$ onto $X$.

Suppose $\nu$ is a regular Borel measure whose support is $X$. Let $\phi$ be the positive linear functional on $\{f \circ g: f \in C(X)\}$ defined by $\phi(f \circ g)=\nu(f)$. Then by [14], p. 20, $\phi$ has a positive extension $\psi$ to all of $C(P)$. Let $\mu$ denote the regular Borel measure that corresponds to $\psi$. Then for each Borel set $A$ in $X, \nu(A)=\mu\left(g^{-1}(A)\right)$. Let $U$ be a nonempty open set on $P$. The proof of Lemma 4.1 shows that $g^{-1}(X \backslash g(P \backslash U)) \subseteq U$ and if $U \neq P$, then $V=X \backslash g(P \backslash U)$ is nonempty. 
Hence, $\mu\left(g^{-1}(V)\right)=\nu(V)>0$. It follows that the support of $\mu$ is all of $P$.

REMARK. Suppose $\mu$ and $\nu$ are related by $\nu(A)=\mu\left(g^{-1}(A)\right)$ for all Borel sets $A$ in $X$. If $\nu$ is nonatomic and $p$ is in $P$, then $\nu(g(p))=0$, and, thus, $0 \leqq \mu(p) \leqq \mu\left(g^{-1}(g(p))\right)=\nu(g(p))=0$ and $\mu$ is nonatomic.

Definition 4.7. If $Y$ is a compact Hausdorff space and $m$ is a regular Borel measure on $Y$, then $m$ is called a normal measure on $Y$ if and only if for each meager Borel subset $A$ of $Y, m(A)=0$.

This concept applied to extremally disconnected spaces is equivalent to Dixmier's notation of normal measure (see [3]).

LEMMA 4.8. If $m$ is a normal measure on $X$, then $m$ restricted to $R(X)$ is a measure on $R(X)$.

Proof. It suffices to show that $m$ is countably additive on $R(X)$. If $U_{1}, U_{2}, \cdots$ is a pairwise disjoint sequence of regular open sets, the least upper bound of this sequence in $R(X)$ is int $c l\left(\bigcup_{n=1}^{\infty} U_{n}\right)$. Since the boundary of an open set is nowhere dense,

$$
m\left(\bigcup_{n=1}^{\infty} U_{n}\right)=m\left(\operatorname{cl} \bigcup_{n=1}^{\infty} U_{n}\right)
$$

and it follows from 2.2 that $m$ (int $\left.\mathrm{cl} \bigcup_{n=1}^{\infty} U_{n}\right)=m\left(\operatorname{cl} \bigcup_{n=1}^{\infty} U_{n}\right)$. Thus, the countable additivity on $R(X)$ follows from the countable additivity on the sigma-algebra of Borel sets.

THEOREM 4.9. If $m$ is a normal measure on $X$, then there is a unique normal measure $m^{\prime}$ on $P$ such that for each Borel subset $E$ of $X, m(E)=m^{\prime}\left(g^{-1}[E]\right)$.

Proof. By 4.8, $m$ defines a measure on $R(X)$. Let $h$ be the isomorphism in 4.5 of $R(X)$ onto $R(P)$, let $i$ be the isomorphism of $R(P)$ onto $B_{0}(P) / I(P)$, and let $j$ be the natural homomorphism of $B_{0}(P)$ onto $B_{0}(P) / I(P)$. For each Borel subset $F$ or $P$, let $m^{\prime}(F)=$ $m\left(h^{-1} i^{-1} j\right)(F)$. Clearly, $m^{\prime}(F)=0$ whenever $F$ is a meager Borel subset of $P$. For each Borel subset $E$ of $X$, let $f(E)=g^{-1}(E)$. By elementary properties of functions (see [12], p. 11), $f$ is an isomorphism of $B_{0}(X)$ onto $B_{0}(P)$. If $E$ is a Borel subset of $X$, then $E$ is equivalent modulo $I(X)$ to a regular open subset $U$ of $X$. Since the symmetric difference $U+E$ is in $I(X)$, by (4.4), $f(E+U)=f(E)+f(U)$ is in $I(P)$, and since $h(U)=\operatorname{cl} f(U), h(U)$ is equivalent to $f(E)$ modulo $I(P)$. Hence, $j[f(E)]$ is the equivalence class of $h(U)$ and 


$$
m^{\prime}[f(E)]=m\left(h^{-1} i^{-1} j f\right)(E)=m(U)=m(E) .
$$

It remains to show that $m^{\prime}$ is a regular Borel measure. Suppose $e$ is a positive real number and $F$ is a Borel subset of $P$. There is a clopen set $C$ such that the symmetric difference $C+F$ is meager. Hence, $F C$ is meager, and there are nowhere dense Borel sets $D_{1}, D_{2}, \cdots$ such that $F \backslash C=\bigcup_{n=1}^{\infty} D_{n}$. Now clearly,

$$
g\left(C \cup \bigcup_{n=1}^{\infty} \operatorname{cl} D_{n}\right)=g(C) \cup \bigcup_{n=1}^{\infty} g\left(\operatorname{cl} D_{n}\right)=E
$$

is a Borel subset of $X$, and $m(E)=m[$ int $g(C)]=m^{\prime}(C)$. Since $m$ is a regular Borel measure, there is an open set $U$ containing $E$ such that $m(U)<m(E)+e$. Thus,

$$
\begin{aligned}
m^{\prime}(F) & =m^{\prime}(C \cup F \backslash C)=m^{\prime}\left(C \cup \bigcup_{n=1}^{\infty} \operatorname{cl} D\right) \leqq m^{\prime}\left[g^{-1}(E)\right]=m(E) \\
& =m^{\prime}(C) \leqq m(U)=m^{\prime}\left[g^{-1}(U)\right]<m(E)+e=m^{\prime}(E)+e .
\end{aligned}
$$

Since $U$ contains

$$
g(C) \cup g(F \backslash C)=g(C \cup F \backslash C)=g(C \cup F),
$$

$g^{-1}(U)$ contain $F$. The uniqueness follows from the fact that the sigma-algebra of Baire subsets of $P$ is generated by clopen subsets (see [12], p. 999). If $m^{\prime \prime}\left[g^{-1}(E)\right]=m(E)$ for each Borel set $E$, then $m^{\prime \prime}$ agrees with $m^{\prime}$ on the clopen subsets of $P$, and hence on the Baire subsets of $P$. By [11], p. 239, the regular Borel extension of $m^{\prime}$ restricted to the Baire subsets is unique.

CoRollary 4.10. There is a one-to-one, order and norm preserving correspondence between the collection $N(X)$ of normal measures on $X$ and the collection $N(P)$ of normal measures on $P$.

Proof. The mapping $g$ of $P$ onto $X$ defines a continuous linear norm decreasing mapping $\hat{g}$ of $M(P)$ onto $M(X)$ such that for each Borel subset $E$ of $X, \hat{g}(m)(E)=m\left[g^{-1}(E)\right]$ (see [15], p. 180). If $\hat{g}$ is restricted to $N(P)$, then the correspondence of the preceding theorem shows that $\hat{g}$ maps $N(P)$ one-to-one, onto $N(X)$. The preservation of order and norm is immediate.

COROLlaRY 4.11. There is a one-to-one, order and norm preserving correspondence between the collection of normal measures whose support is $X$ and the collection of normal measures whose support is $P$.

Proof. Let $\hat{g}$ be the mapping defined in the previous corollary. 
If $m$ is a normal measure whose support is $P$, then $\hat{g}(m)$ is a normal measure. If $U$ is a nonempty open subset of $X$, then $g^{-1}(U)$ is a nonempty open subset of $P$ and $\hat{g}(m)(U)=m\left[g^{-1}(U)\right]>0$. If $m$ is a normal measure on $X$ and $U$ is a nonempty open subset of $P$, then $U$ contains a nonempty clopen set $C$ and by (4.2), $\operatorname{cl}^{-1}[$ int $g(C)]=C$. Thus, $g^{-1}(m)(U) \geqq g^{-1}(m)(C) \geqq g^{-1}(m)\left[g^{-1}\right.$ int $\left.\{g(C)\}\right]=m[$ int $g(C)]>0$.

Recall that each signed regular Borel measure $m$ on a compact Hausdorff space $Y$ may be written as $m=m^{+}-m^{-}$. If $Y$ is a compact Hausdorff space and $m_{1}$ and $m_{2}$ are normal measures on $Y$, then $m=m_{1}-m_{2}$ is a signed regular Borel measure on $Y$, and since $m_{1} \geqq m^{+} \geqq 0$ and $m_{2} \geqq m^{-} \geqq 0, m^{+}$and $m^{-}$are also normal measures on $Y$. Thus, $\{m: m$ is a signed regular Borel measure on $Y$ such that $m^{+}$and $m^{-}$are normal $\}=\left\{m: m=m_{1}-m_{2}\right.$ where $m_{1}$ and $m_{2}$ are normal measures on $Y$. This collection is called the collection of signed normal measures on $Y$ and is denoted by $M^{\prime}(Y)$. It is easy to show that $M^{\prime}(Y)$ is a closed linear subspace $M(Y)$ for each compact Hausdorff space $Y$. It also follows easily from 4.10 that $M^{\prime}(X)$ and $M^{\prime}(P)$ are linearly isometric. For a different proof of this, see [2].

The following theorem summarizes some of the results in this section in combination with some results from the theory of measure algebras.

THEOREM 4.12. The following statements are equivalent.

(i) There is a normal measure whose support is $X$.

(ii) There is a normal measure whose support is $P$.

(iii) There is a strictly positive (countably additive) measure of $R(X)$ (resp. $R(P)$ ).

(iv) There is a regular Borel measure on $P$ whose support is $P$ and every meager set in $P$ is nowhere dense.

(v) There is a regular Borel measure on $X$ whose support is $X$ and every meager set in $X$ is nowhere dense.

Proof. Theorem 4.6 and 4.9 yield that (i) is equivalent to (ii). Since $R(P), R(X), B_{0}(X) / I(X)$, and $B_{0}(P) / I(P)$ are all isomorphic under an isomorphism that preserves countable operations, (i) equivalent to (iii) and (ii) is equivalent to (iii). Suppose there is a normal measure $\mu$ on $P$ with support $P$. Let $B \subset P$ be a meager set. Then $\mu(B)=$ $\mu$ (int $\mathrm{cl} B$ ) and hence $B$ is nowhere dense (see [3], p. 158). Thus (ii) implies (iv).

Suppose (iv) is true. Then by Theorem 4.6 there is a regular Borel measure whose support is $X$. If $B \subset X$ is meager, then by Lemma $4.4 g^{-1}(B)$ is meager and thus $g^{-1}(B)$ is nowhere dense. Hence, by Lemma 4.4 again, $B$ is nowhere dense and (iv) implies (v). A 
similar argument shows that (v) implies (iv). For, by Theorem 4.6 again, if there is a regular Borel measure whose support is $X$, then there is one whose support is $P$. Suppose $B \subset P$ is closed and nowhere dense. Then $g(B)$ is closed and nowhere dense. Thus, if $B \subset P$ is meager, then $g(B)$ is meager. It follows that if each meager set in $X$ is nowhere dense, then each meager set in $P$ is nowhere dense. Finally, (iv) implies (iii). For, in [13] Kelley shows that if a complete Boolean algebra $A$ has a strictly positive finitely additive measure and if each meager set in the Stone space of $A$ in nowhere dense, then $A$ has a strictly positive countably additive measure. Thus, if $\mu$ is a regular Borel measure whose support is $P$ and each meager set in $P$ is nowhere dense, then $\mu$ induces a finitely additive strictly positive measure on $R(P), R(P)$ is a complete Boolean algebra, the Stone space of $R(P)$ is $P$, and by the above cited result, $R(P)$ has a countably additive strictly positive measure.

\section{BIBLIOGRAPHY}

1. G. Birkhoff, Lattice theory, Am. Math. Soc. Colloquium Publications, Vol. 25, Providence, Rhode Island, 1961.

2. H. B. Cohen and H. E. Lacey, On injective envelopes of Banach spaces, Proceedings of the Conference on Projections and Related Topics, Clemson University, 1968, pp. 27-40. To appear in J. Final. Anal.

3. J. Dixmier, Sur certains espaces consideres par M. H. Stone, Sum. Bras. Math. 2 (1951), 151-181.

4. N. Dunford and J. T. Schwartz, Linear operator, Part I, Interscience Publishers, Inc., New York, 1964.

5. D. A. Edwards, A class of topological Boolean algebras, Proc. Lon. Math. Soc. (3) 13 (1963), 413-429.

6. R. E. Edwards, Functional analysis, Holt, Rinehart and Winston, New York, 1965.

7. H. Gaifman, Concerning measures on Boolean algebras, Pacific J. Math. 14 (1964), 61-73.

8. A. M. Gleason, Projective topological spaces, Illinois J. Math. 2 (1958), 482-489.

9. A. Grothendieck, Une characterization vectoriellemetrique des Espaces $L^{1}$, Canad. J. Math. 7 (1955), 552-561.

10. P. R. Halmos, Lectures on Boolean algebras, D. Van Nostrand Co., Inc., Princeton, N. J., 1963.

11. Measure theory, D. Van Nostrand Co., Inc., Princeton, N. J., 1950.

12. J. L. Kelley, General topology, D. Van Nostrand Co., Inc., Princeton, N. J., 1955.

13_, Measures on Boolean algebras, Pacific J. Math. 9 (1959), 1165-1176.

14. J. L. Kelley, I. Namioka, and co-authors, Linear topological spaces, D. Van Nostrand Co., Inc., Princeton, N. J., 1963.

15. V. L. Klee, Jr., Convex sets in linear spaces, Duke Math. J. 18 (1951), 443-466.

16. D. Maharam, An algebraic characterization of measure algebras, Ann. of Math. 48 (1947), 154-167.

17. A. Pelczynski and Z. Semadeni, Spaces of continuous functions III, Studia Math. 18 (1959), 211-222.

18. R. Sikorski, Boolean algebras, second edition, Academic Press. Inc., New York, 1964. 
Received July 19, 1967. The second author held a Resident Post-Doctoral Research Associateship at NASA-MSC Houston, Texas, during the preparation of part of this paper.

University of TeXas at Austin 


\section{PACIFIC JOURNAL OF MATHEMATICS}

EDITORS

H. ROYDEN

Stanford University

Stanford, California

R. R. Phelps

University of Washington

Seattle, Washington 98105
J. DugundJI

Department of Mathematics

University of Southern California

Los Angeles, California 90007

\section{RICHARD ARENS}

University of California

Los Angeles, California 90024

\section{ASSOCIATE EDITORS}
E. F. BeCKENBACH
B. H. NeUmanN
F. WOLF
K. YOSIDA

\section{SUPPORTING INSTITUTIONS}

UNIVERSITY OF BRITISH COLUMBIA CALIFORNIA INSTITUTE OF TECHNOLOGY UNIVERSITY OF CALIFORNIA MONTANA STATE UNIVERSITY UNIVERSITY OF NEVADA NEW MEXICO STATE UNIVERSITY OREGON STATE UNIVERSITY UNIVERSITY OF OREGON OSAKA UNIVERSITY UNIVERSITY OF SOUTHERN CALIFORNIA
STANFORD UNIVERSITY UNIVERSITY OF TOKYO UNIVERSITY OF UTAH WASHINGTON STATE UNIVERSITY UNIVERSITY OF WASHINGTON

AMERICAN MATHEMATICAL SOCIETY CHEVRON RESEARCH CORPORATION TRW SYSTEMS NAVAL WEAPONS CENTER 


\section{Pacific Journal of Mathematics \\ Vol. 27, No. $1 \quad$ January, 1968}

Willard Ellis Baxter, On rings with proper involution ............... 1

Donald John Charles Bures, Tensor products of $W^{*}$-algebras........... 13

James Calvert, Integral inequalities involving second order derivatives . . . . 39

Edward Dewey Davis, Further remarks on ideals of the principal class.... 49

Le Baron O. Ferguson, Uniform approximation by polynomials with integral

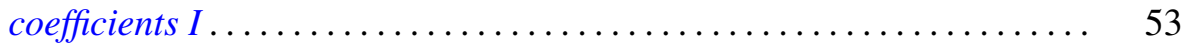

Francis James Flanigan, Algebraic geography: Varieties of structure

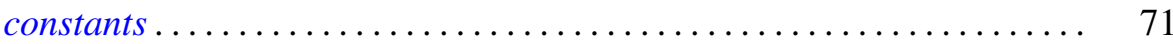

Denis Ragan Floyd, On QF -1 algebras ..................... 81

David Scott Geiger, Closed systems of functions and predicates ......... 95

Delma Joseph Hebert, Jr. and Howard E. Lacey, On supports of regular Borel measures ................................... 101

Martin Edward Price, On the variation of the Bernstein polynomials of a function of unbounded variation ........................ 119

Louise Arakelian Raphael, On a characterization of infinite complex matrices mapping the space of analytic sequences into itself........ 123

Louis Jackson Ratliff, Jr., A characterization of analytically unramified

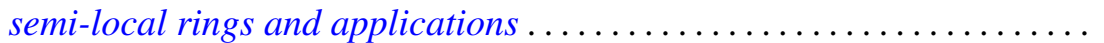

S. A. E. Sherif, A Tauberian relation between the Borel and the Lototsky transforms of series ................................ 145

Robert C. Sine, Geometric theory of a single Markov operator .......... 155

Armond E. Spencer, Maximal nonnormal chains in finite groups......... 167

Li Pi Su, Algebraic properties of certain rings of continuous functions .... 175

G. P. Szegô, A theorem of Rolle's type in $E^{n}$ for functions of the class $C^{1} \ldots 193$

Giovanni Viglino, A co-topological application to minimal spaces ........ 197

B. R. Wenner, Dimension on boundaries of $\varepsilon$-spheres ............... 201 